\title{
Ectasia of the left atrial appendage
}

INSERM

\section{Source}

INSERM. (1999). Orphanet: an online rare disease and orphan drug data base. Ectasia of the left atrial appendage. ORPHA:99102

Ectasia of the left atrial appendage is a rare cardiac malformation characterized by the enlargement of the left auricle without any other associated cardiac lesions. It can be asymptomatic (discovered fortuitously during routine chest imaging as an unusual cardiac shadow) or present clinically with supraventricular tachyarrhythmia, paroxysmal tachycardia, embolic events, respiratory distress, chest pain, ang ina pectoris or heart failure. 